\title{
A Knock-In Model of Human Epilepsy in Drosophila Reveals a Novel Cellular Mechanism Associated with Heat-Induced Seizure
}

\author{
Lei Sun, ${ }^{1}$ Jeff Gilligan, ${ }^{2}$ Cynthia Staber, ${ }^{2}$ Ryan J. Schutte, ${ }^{1}$ Vivian Nguyen, ${ }^{1}$ Diane K. 0 'Dowd, ${ }^{1 \star}$ and Robert Reenan ${ }^{2 \star}$ \\ ${ }^{1}$ Department of Anatomy and Neurobiology; Developmental and Cell Biology, University of California, Irvine, Irvine, California 92697-1280, and \\ ${ }^{2}$ Department of Molecular Biology, Cell Biology, and Biochemistry, Brown University, Providence, Rhode Island 02912
}

Over 40 missense mutations in the human SCN1A sodium channel gene are linked to an epilepsy syndrome termed genetic epilepsy with febrile seizures plus (GEFS +). Inheritance of GEFS + is dominant, but the underlying cellular mechanisms remain poorly understood. Here we report that knock-in of a GEFS + SCN1A mutation (K1270T) into the Drosophila sodium channel gene, para, causes a semidominant temperature-induced seizure phenotype. Electrophysiological studies of GABAergic interneurons in the brains of adult GEFS+ flies reveal a novel cellular mechanism underlying heat-induced seizures: the deactivation threshold for persistent sodium currents reversibly shifts to a more negative voltage when the temperature is elevated. This leads to sustained depolarizations in GABAergic neurons and reduced inhibitory activity in the central nervous system. Furthermore, our data indicate a natural temperature-dependent shift in sodium current deactivation (exacerbated by mutation) may contribute to febrile seizures in GEFS + and perhaps normal individuals.

\section{Introduction}

Many human seizure disorders are caused by mutations in ion channel genes. The SCN1A sodium channel gene alone has in excess of 600 mutations conferring a wide spectrum of epilepsies (Claes et al., 2009; Lossin, 2009; Catterall et al., 2010). Truncating and presumed null mutations of SCN1A are associated with severe disorders, such as Dravet syndrome (DS). More conservative missense mutations generally confer more benign conditions, including $>40$ that result in genetic epilepsy with febrile seizures plus (GEFS + ), which is characterized by febrile seizures that persist in individuals past 6 years of age (Scheffer and Berkovic, 1997) (http://www.molgen.vib-ua.be/ SCN1AMutations/Mutations/Default.cfm). The cellular mechanisms underlying seizure disorders associated with this broad array of mutations are not well understood.

Analysis of a number of SCN1A mutations in heterologous expression systems has revealed a variety of biophysical changes in sodium channel function that could potentially lead to seizure phenotypes, but it is unclear whether these are reflective of alter-

\footnotetext{
Received June 19, 2012; revised July 25, 2012; accepted Aug. 1, 2012.

Author contributions: L.S., J.G., C.S., R.J.S., D.K.O., and R.R. designed research; L.S., J.G., C.S., R.J.S., V.N., D.K.O., and R.R. performed research; L.S., R.J.S., V.N., D.K.O., and R.R. analyzed data; L.S., R.J.S., D.K.O., and R.R. wrote the paper.

This work was supported by NIH Grant NS27501 and a grant from the Howard Hughes Medical Institute Professor Program (D.K.O.). Support was also provided by NIH Grant NS074686 and an Ellison Medical Foundation Senior Scholar Award (R.R.).

${ }^{*}$ D.K.O. and R.R. contributed equally to this work.

The authors declare no competing financial interests.

Correspondence should be addressed to either of the following: Diane 0'Dowd, 112 Irvine Hall, University of California, Irvine, Irvine, CA 92697-1280, E-mail: dkodowd@uci.edu; or Robert Reenan, 372 Sidney E. Frank Hall Life Sciences Building, Brown University, Providence, RI 02912. E-mail: robert_reenan@brown.edu.

DOI:10.1523/JNEUROSCI.2932-12.2012

Copyright $\odot 2012$ the authors $\quad 0270-6474 / 12 / 3214145-11 \$ 15.00 / 0$
}

ations that occur in neurons (Lossin et al., 2002, 2003; Escayg and Goldin, 2010). Recent results in DS and GEFS+ knock-in mouse models demonstrated reduced activity in GABAergic inhibitory neurons examined at room temperature (Ogiwara et al., 2007; Martin et al., 2010). However, the underlying changes in sodium channel function were different in these SCN1A mutants, and neither channel function nor neuronal excitability was explored at high temperature. Using transgenic mice to assess channel defects for even a fraction of the known SCN1A mutations would be very resource intensive.

Drosophila is another genetically tractable animal model that has been used to study seizure disorders (Song and Tanouye, 2008). A classic forward genetic approach has identified a number of mutants with altered seizure sensitivity but none that result from mutations homologous to seizure-causing SCN1A mutations in humans (Royden et al., 1987; Pavlidis et al., 1994; Zhang et al., 2002; Fergestad et al., 2006; Parker et al., 2011). Recent technical advances have now made it feasible to readily target and replace endogenous sequences in the fly genome using homologous recombination (Rong and Golic, 2000; Rong et al., 2002; Staber et al., 2011). Knock-in of specific disease causing mutations into the fly genome has the potential to provide a rapid and low-cost platform for studying the cellular mechanisms of heritable human diseases. In addition, knock-in flies can be used in combination with forward genetic screens to identify suppressor and/or enhancer mutations, a strategy that is challenging in humans and rodent models but well established in Drosophila (Song et al., 2007, 2008).

In this study, a disease-causing GEFS + mutation (SCN1A, K1270T) (Abou-Khalil et al., 2001) was knocked into the Drosophila para sodium channel gene at the comparable position, conferring a semidominant temperature-sensitive seizure phe- 


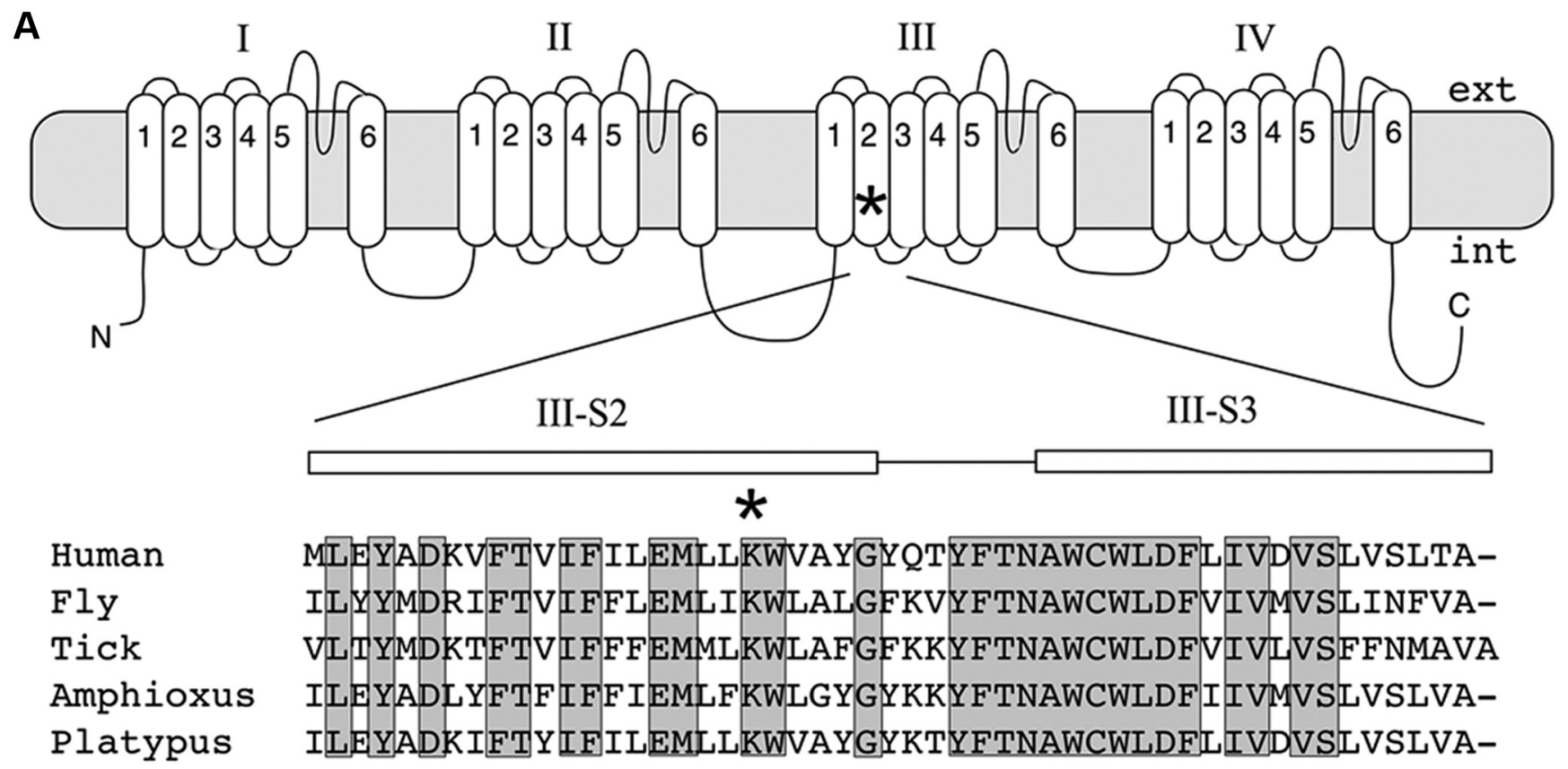

B

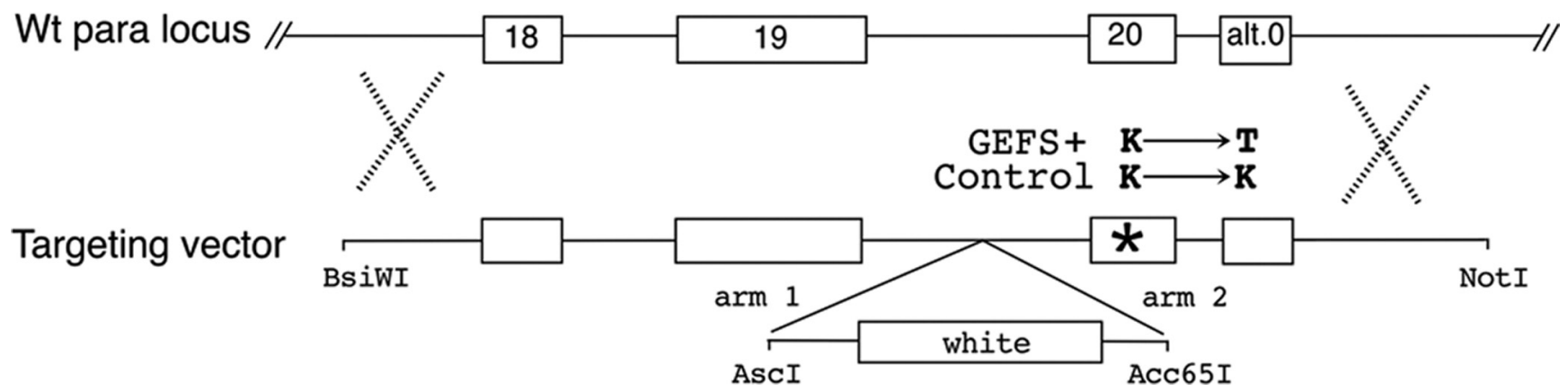

Figure 1. Generation of GEFS + and control flies by targeted knock-in. A, Schematic diagram of a sodium channel $\alpha$-subunit. Lysine residue altered in the human K1270T GEFS + mutation is located in the second transmembrane segment in homology domain III (indicated by an asterisk). Comparison of the amino acid sequence in this region reveals a high degree of homology between species. Identical amino acids are shaded gray. Location of lysine residue altered in mutants is indicated by the asterisk. $\boldsymbol{B}$, Diagram of the wild-type para sodium channel locus and the targeting vectors. The GEFS + targeting vector contained the $\mathrm{K}$ to $\mathrm{T}$ substitution, and the control targeting vector contained the control substitution $\mathrm{K}$ to $\mathrm{K}$. Location of the substitution is indicated by an asterisk. Homologous recombination events between incoming linear recombinogenic DNA and endogenous para locus are indicated by crossed lines.

notype. Electrophysiological recordings from GABAergic neurons in brains of adult mutant flies at both permissive and high temperature revealed a temperature-dependent alteration in sodium currents. This represents a novel mechanism for altering inhibitory activity contributing to heat induced seizure.

\section{Materials and Methods}

Ends-out homologous recombination of the para locus. To introduce the K1270T mutation into the para sodium channel gene in Drosophila we performed ends-out homologous recombination using a similar methodology to that reported previously (Maggert et al., 2008) with modifications (Staber et al., 2011). Briefly, we used the ends-out targeting vector $\mathrm{p}[\mathrm{w} 25.2]$, which contains the white $+(w+)$ selectable eye-color minigene flanked by LoxP sites allowing for subsequent removal by Cre recombinase. Homology arms were cloned and sequence verified in pTOPO (Invitrogen). The mutation analogous to GEFS + K1270T was introduced into arm 2 using the QuickChange mutagenesis kit (Stratagene) and the following mutagenic primers: GEFS+ F, 5'-GGTTATATTCTTCTTGGA AATGTTAATCACCTGGTTGGCGCTCGGCTTCAAAGTG-3'; GEFS+ R, 5' -CACTTTGAAGCCGAGCGCCAACCAGGTGATTAACATTTCC AAGAAGAATATAACC- $3^{\prime}$.
Mutagenized clones were resequenced to insure that only the desired mutation was introduced. Homology arms 1 and 2 were cloned sequentially into the multiple cloning sites of the vector to generate $\mathrm{p}$ [w25paraK1270T], which was then introduced randomly into the Drosophila genome by standard transgenic methods (Genetic Services).

In parallel, a wild-type substitution, $\mathrm{K} 1270 \mathrm{~K}$, was introduced using an identical procedure to generate $\mathrm{p}$ [w25-paraK1270K], which was used to create a transgenic control line in the same genetic background.

Details of the cloning strategy were as follows, where all genomic coordinates are given by the Drosophila melanogaster draft (Berkeley Drosophila Genome Project Release 5) with release 5.12 annotation provided by Flybase at the University of California Santa Cruz Genome Browser (http://genome.ucsc.edu). Arm 1 is the $5^{\prime}$ arm of p[w25-para] (Fig. 1 B) and was generated by PCR amplification to incorporate cloning sites as follows: Arm 1, BsiWI-16,378,805-16,376,207-AscI. Arm 2 is the 3' arm of $\mathrm{p}$ [w25-para] and was generated by PCR amplification to incorporate cloning sites as follows: Arm 2, Acc65I-16,376,130-16,373,532-NotI.

Targeting was performed to generate multiple independent targeting events of $\mathrm{p}[\mathrm{w} 25$-paraWT $]$ and $\mathrm{p}[\mathrm{w} 25$-paraGEFS +$]$ starting from independent transgenic insertions. Targeted alleles were validated for correct homologous recombination to the para locus by amplification using 
primers outside the region of targeting to sequences specific to the unique $w+$ selectable marker. Amplicons of targeted alleles were sequenced to verify only the presence of indicated wild-type sequences (or GEFS + mutation). Subsequent removal of the $w+$ minigene selectable marker was achieved by performing crosses to animals expressing Cre recombinase and reisolation of targeted chromosomes containing a single LoxP site. PCR amplification and sequencing across the post-CRE allele was performed to validate the accuracy of the removal of white sequences.

Fly lines. The recombinant alleles, both the mutant and control substitution, were subsequently backcrossed to the w1118 stocks, a whiteeyed genetic background, for five generations. Finally, these were crossed to a stock with UAS-GFP, $w+$ on the second chromosome. Flies homozygous for the recombinant alleles on the $\mathrm{X}$ chromosome and the UASGFP, $w+$ on the second chromosome were selected and used to establish the two strains used in all experiments reported. The homozygous mutant $(\mathrm{K} 1270 \mathrm{~T})$ and control (K1270K) strains are referred to as GEFS+ and control. Heterozygotes were generated by crossing GEFS + mutant and control flies.

Heat-induced seizure assay. Two-day-old flies grown at room temperature $\left(22-24^{\circ} \mathrm{C}\right)$ were isolated in individual plastic vials. Flies were allowed to acclimate to the new environment for 5-15 min before the vial was immersed in a water bath of $40^{\circ} \mathrm{C}$ for $2 \mathrm{~min}$. Seizures were defined as a period of brief leg twitches, followed by failure to maintain standing posture, with wing flapping, leg twitching, and sometimes abdominal curling. The status of individual flies (seizing or not seizing) was determined at $5 \mathrm{~s}$ intervals, and the probability of seizing at each time point was calculated for comparison. All tests were performed blind with respect to fly genotype.

Whole-cell recordings from local neurons in isolated whole brain. All brains were obtained from adult male GEFS + or control flies ( $2 \mathrm{~d}$ old). The entire brain was removed from the head and prepared for recordings as described previously (Gu and O'Dowd, 2006, 2007). Pipettes were visually targeted to a small subset of local neurons (LNs) in an anatomically distinct region of the dorsal lateral aspect of the antenna lobe. Whole-cell sodium currents, depolarization-evoked action potentials, and spontaneous bursts of firing were recorded with standard whole-cell pipettes of 10-11 M $\Omega$. All voltages reported refer to pipette potentials at the soma.

Isolated sodium currents were recorded using a pipette solution containing the following (in $\mathrm{mm}$ ): $102 \mathrm{D}$-gluconic acid, $102 \mathrm{CsOH}, 0.085$ $\mathrm{CaCl}_{2}, 1.7 \mathrm{MgCl}_{2}, 17 \mathrm{NaCl}, 0.94 \mathrm{EGTA}, 8.5$ HEPES, and 4.5 ATP. The $\mathrm{pH}$ was adjusted to 7.2 and osmolarity to $235 \mathrm{mOsm}$. The external solution contained the following (in mM): $120 \mathrm{NaCl}, 1.8 \mathrm{CoCl}_{2}, 0.8 \mathrm{MgCl}_{2}, 3 \mathrm{KCl}$, 5 glucose, 10 HEPES, 2.5 tetraethylammonium (TEA), and 1.0 4-aminopyridine (4-AP). It also contained the synaptic receptor blockers D-tubocurarine (curarine, $20 \mu \mathrm{M}$ ) and picrotoxin (PTX, $10 \mu \mathrm{M}$ ). The $\mathrm{pH}$ was adjusted to 7.2 and osmolarity to $250 \mathrm{mOsm}$. Data shown were corrected for the $5 \mathrm{mV}$ liquid junction potential generated in these solutions. Depolarization-evoked action potentials were recorded using the same internal solution as that used for sodium currents except that cesium gluconate was replaced by potassium gluconate, and there was no TEA or 4-AP. For examination of the evoked firing properties, the membrane potential was held at $-75 \mathrm{mV}$ by injection of hyperpolarizing holding current. Spontaneous burst firing was recorded using the same internal and external solution used for depolarization-evoked action potentials, with the omission of PTX and curarine. In some of the recordings, $0.4 \%$ biocytin was added to internal solution to label LNs.

The chamber was continuously perfused at $1 \mathrm{ml} / \mathrm{min}$ with external solution, and the temperature in the chamber was controlled and monitored using a CL-100 Biopolar Temperature Controller (Harvard Apparatus). Although there were small variations in the heating profile between individual neurons during the 2 min heating period, there was no difference between the genotypes in the maximal temperatures examined (GEFS,$+ 34.7 \pm 1.4^{\circ} \mathrm{C}$; control, $34.7 \pm 1.1^{\circ} \mathrm{C}$, mean $\pm \mathrm{SD}$ ).

Data were acquired with a List EPC7 amplifier, a digidata $1322 \mathrm{~A}$ digital-to-analog converter (Molecular Devices), a Dell computer (Dimension 8200), and pClamp9 software (Molecular Devices).

Fluorescent immunostaining. Dual staining for Nc82 and intracellular biocytin were performed at room temperature. Immediately after whole- cell recording, brains were fixed in 4\% paraformaldehyde in PBS for 2-4 $\mathrm{h}$ at $4^{\circ} \mathrm{C}$, rinsed with PBS, and permeabilized with $0.2 \%$ Triton X-100 in PBS (PBST) for $40 \mathrm{~min}$, followed by $4 \%$ bovine serum albumin/PBST ( 40 $\min$ ). Brains were incubated in primary antibody $(1 \mathrm{~h}), \mathrm{Nc} 82$ (mouse MAB, 1:1000; Developmental Studies Hybridoma Bank), followed by washing for $30 \mathrm{~min}$ in PBST $(3 \times)$. Brains were incubated for $1 \mathrm{~h}$ with fluorescently tagged secondary antibody (goat anti-mouse Alexa Fluor 546 (1:1000; Invitrogen) and streptavidin conjugated to Alexa Fluor 488 (1:1000; Invitrogen) to label biocytin. After incubation, brains were washed for $30 \mathrm{~min}$ in several changes of PBS and mounted in Fluoromount G. Confocal fluorescence microscopy was performed on a Zeiss LSM 510 using a $40 \times$ oil-immersion objective.

For dual staining for GABA and intracellular biocytin, the above procedure was modified to include overnight fixation of brains in $4 \%$ paraformaldehyde. Primary antibody incubation (anti-GABA, mouse MAB, 1:500; Sigma) was for $2 \mathrm{~h}$ at room temperature.

Acute PTX exposure. Two-day-old male GEFS + and control flies were fasted for $6 \mathrm{~h}$ before exposure to PTX (0-0.4 mm; Sigma) in 5\% sucrose solution with food coloring. Flies were fed for $15 \mathrm{~min}$, and only those with food coloring visible in the abdomen were selected for the heatinduced seizure assay. Heat-induced seizures were recorded 5-30 min after feeding. Experiments were conducted blind with respect to PTX concentration and genotype.

Statistics. Comparisons between GEFS + and control were done with two-tailed independent Student's $t$ test (two genotypes) or one-way ANOVA followed by Bonferroni's post hoc test (more than two genotypes). Comparisons between permissive and elevated temperatures within the same genotype were done with paired $t$ test. Differences between mean values were considered significant at $p \leq 0.05$.

\section{Results}

\section{Targeting the GEFS+ mutation using ends-out homologous recombination}

The human GEFS + mutation K1270T (Abou-Khalil et al., 2001) was chosen because it is in the highly conserved homology domain III, transmembrane segment 2 , and the amino acid $\mathrm{K}$ is invariant in all identified SCN1A orthologues (Fig. 1A). The functional consequences of K1270T have not been assessed in either heterologous studies or knock-in mice, thus presenting a potential to uncover novel mechanistic insights into epileptogenesis. Both wild-type K1270K and mutant K1270T targeting constructs were introduced into the Drosophila genome in multiple independent transgenic lines that were used for subsequent homologous recombination (Fig. $1 B$ ). Amplicons of targeted alleles were sequenced to verify the presence of wild-type and mutant sequences. The homozygous mutant (K1270T) and control $(\mathrm{K} 1270 \mathrm{~K})$ strains are referred to as GEFS+ and control.

\section{GEFS + flies exhibit a heat-induced seizure phenotype}

Individual flies were observed in plastic vials for $2 \mathrm{~min}$ following immersion of the vial in a $40^{\circ} \mathrm{C}$ water bath. Since the para gene is located on the $\mathrm{X}$ chromosome, we assessed activity in hemizygous male $(\mathrm{GEFS}+/ \mathrm{Y})$ and homozygous female (GEFS+/GEFS+) mutants separately. Both sexes exhibited an initial period of increased locomotor velocity followed by seizure activity. Seizures began $20-30 \mathrm{~s}$ after immersion in the $40^{\circ} \mathrm{C}$ water bath and once a seizure initiated in a GEFS + mutant, it continued for the remainder of the $2 \mathrm{~min}$ period. The probability of seizing increased as a function of time and by 2 min $>96 \%$ of flies were seizing (Fig. 2). There was no difference between males and females. When vials were removed from the water bath there was an abrupt cessation of activity. The flies remained motionless and were unresponsive to tapping of the vials for varying periods of time before showing brief periods of spontaneous movement, generally in one or two legs. Recovery of the ability to stand and resume walking/climb- 


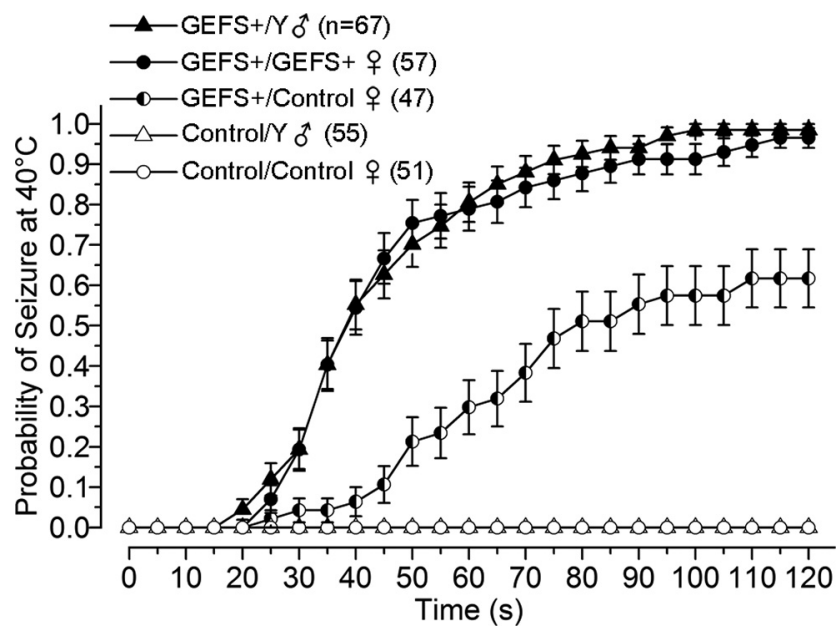

Figure 2. GEFS + flies exhibit a temperature-induced seizure phenotype. Seizures are defined as a brief period of leg twitches, followed by an inability to maintain standing posture, with wing flapping, leg twitching, and sometimes abdomen curling. Individual 2-d-old flies were put into vials that were immersed in a water bath $\left(40^{\circ} \mathrm{C}, 2 \mathrm{~min}\right)$. The status of each fly (seizing or not seizing) was determined at 5 s intervals, and the probability of seizing at each time point was calculated for the population of flies examined in each genotype. The probability of seizing after $2 \mathrm{~min}$ at $40^{\circ} \mathrm{C}$ is significantly different between the mutants (GEFS $+/ \mathrm{Y}$ and GEFS + /GEFS +), heterozygotes (GEFS +/control) and controls (control/Y and control/control; $p<0.001$, ANOVA, Bonferroni's post hoc test). Symbols and error bars represent mean $\pm \mathrm{SEM}$ from the number of flies indicated $(n)$.

ing behavior required 9-28 $\mathrm{min}$ (females, $19.6 \pm 1.3 \mathrm{~min}$; males, $11.2 \pm 0.6 \mathrm{~min})$.

In the control strain, both males and females exhibited increases in locomotor activity when vials were immersed in a $40^{\circ} \mathrm{C}$ water bath. However, there was no evidence of seizures during the 2 min immersion (Fig. 2). In addition, there was no cessation of activity when the vials were removed from the water bath.

Since the human disease is dominantly inherited, we tested the temperature-sensitive behavior of heterozygous females (GEFS+/ control). The time to seizure onset in GEFS+/control was delayed, and the maximal probability of seizing was less than in the homozygous GEFS+ mutants (Fig. 2). Furthermore, there was heterogeneity in the response of the heterozygotes with $21 \%$ showing no seizures. The majority (79\%) did exhibit seizure activity, but this could start and stop more than once during the 2 min period. When vials were removed from the water bath, $46 \%$ of the seizing flies exhibited abrupt cessation of movement. Recovery of the ability to stand and resume normal locomotion required $7-20 \mathrm{~min}$ (mean, $12.3 \pm 2.5 \mathrm{~min}$ ). The remainder of the flies resumed normal locomotion in less than a minute following removal of the vial from the water bath.

The probability of seizing after $2 \mathrm{~min}$ at $40^{\circ} \mathrm{C}$ was significantly different, with homozygotes higher than heterozygotes, and heterozygotes higher than control $(p<0.001$, ANOVA, Bonferroni's post hoc test). These data indicate that the GEFS+ K1270T mutation in Drosophila is semidominant with variable penetrance.

\section{Alterations in sodium currents in GEFS + GABAergic LNs at elevated temperature}

To examine the underlying mechanism of temperature sensitive seizures caused by the K1270T mutation in sodium channels, we focused our initial study on GABAergic LNs in the isolated adult brain preparation of adult male flies (Gu and O'Dowd, 2006, 2007). The LNs examined were in the dorsal lateral aspect of the antennal lobe and the majority (32 of 35) were pan-glomerular
(Chou et al., 2010), projecting to the entire ipsilateral antennal lobe (Fig. 3A). Immunostaining showed most LNs in this region were GABAergic, and biocytin-filled neurons were colabeled with anti-GABA antibodies ( $n=4$ of 4 ; data not shown).

Isolated sodium currents in all LNs exhibited two distinct components: a large-amplitude transient current $\left(I_{\mathrm{NaT}}\right)$ and a smalleramplitude persistent current $\left(I_{\mathrm{NaP}}\right)$ (Fig. $3 B$ ). The sodium currents were not well space clamped in either genotype. This is consistent with most channels being located in neuronal processes, electrotonically distant from the cell body. While this precluded an accurate biophysical description of the current, comparison of current properties was used to identify differences associated with genotype and/or response to elevated temperature.

The $I_{\mathrm{NaT}}$ amplitudes were not significantly different in GEFS + and control LNs at $23^{\circ} \mathrm{C}$, and elevation of the temperature did not significantly affect $I_{\mathrm{NaT}}$ amplitude in either genotype (Fig. 3C). The threshold voltage for activating sodium currents was significantly more hyperpolarized in GEFS + compared to control LNs at both 23 and $35^{\circ} \mathrm{C}$ (Fig. 3D). However, the activation threshold did not change at elevated temperature in either control or GEFS+.

In contrast, there were both temperature- and genotypespecific differences in the properties of $I_{\mathrm{NaP}}$. At elevated temperature, the $I_{\mathrm{NaP}}$ often failed to deactivate when the depolarizing voltage step was terminated (Fig. $3 C$, arrow). This was seen only at high temperature and was more frequent in GEFS + ( 8 of 17) compared to control ( 3 of 18 ) neurons, thus identifying a potential alteration that could contribute to the temperature-induced seizure phenotype.

A three-step protocol was used to further examine the voltage dependence of $I_{\mathrm{NaP}}$ deactivation (Fig. $4 A, B$ ). A prepulse to inactivate $I_{\mathrm{NaT}}$ was followed by a family of test pulses to increasingly hyperpolarized potentials. The threshold voltage for $I_{\mathrm{NaP}}$ deactivation was defined as the most depolarized test step that resulted in current deactivation. For the control LN shown the deactivation threshold was $-45 \mathrm{mV}$ at $23^{\circ} \mathrm{C}$. When the temperature was raised to $35^{\circ} \mathrm{C}$, the voltage for $I_{\mathrm{NaP}}$ deactivation shifted by -10 $\mathrm{mV}$, from -45 to $-55 \mathrm{mV}$ (Fig. $4 A$ ).

In a typical GEFS $+\mathrm{LN}$, at $23^{\circ} \mathrm{C}$, the deactivation threshold for the $I_{\mathrm{NaP}}$ was $-55 \mathrm{mV}$ (Fig. $4 \mathrm{~B}$ ). When the temperature was raised to $35^{\circ} \mathrm{C}$, there was a larger shift in current deactivation voltage, from -55 to $-75 \mathrm{mV}$ (Fig. $4 \mathrm{~B}$ ). The average threshold for deactivation at $35^{\circ} \mathrm{C}$ in GEFS + was significantly more hyperpolarized than control (Fig. $4 C)(p<0.05)$. There was no significant difference in deactivation voltage of $I_{\mathrm{NaP}}$ in control and GEFS + LNs at $23^{\circ} \mathrm{C}$ (Fig. $\left.4 C\right)(p=0.19)$. These data indicate that the GEFS + mutation causes a significantly larger temperature-dependent hyperpolarizing shift in the deactivation voltage of the $I_{\mathrm{NaP}}$.

In both GEFS + and control LNs, the amplitude of the $I_{\mathrm{NaP}}$ exhibited a change with test potential that was well fit by a linear regression at both 23 and $35^{\circ} \mathrm{C}$ (Fig. $4 A i, B i$ ). The slope of $I-V$ curve, equal to the conductance of the $I_{\mathrm{NaP}}\left[I=g\left(V-V_{\mathrm{R}}\right)\right]$, was steeper at $35^{\circ} \mathrm{C}$ for both genotypes (Fig. $4 \mathrm{Ai}$ ). However, the conductance was not different between GEFS + and control at either 23 or $35^{\circ} \mathrm{C}$ (Fig. $4 \mathrm{D}$ ). These data suggest that the GEFS+ mutation does not affect the magnitude or the temperature-dependent increase in conductance of $I_{\mathrm{NaP}}$.

In GEFS + neurons, the larger repolarization required to deactivate the $I_{\mathrm{NaP}}$ at elevated temperature, along with the more hyperpolarized activation threshold for the sodium current, results in sodium currents that are active over a greater voltage range (Fig. $4 E$ ) 
A

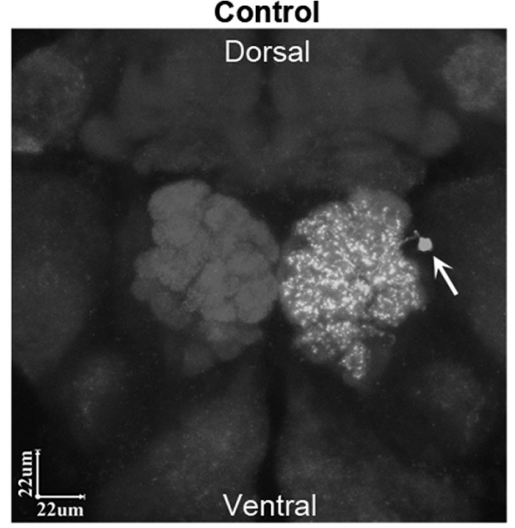
B Control
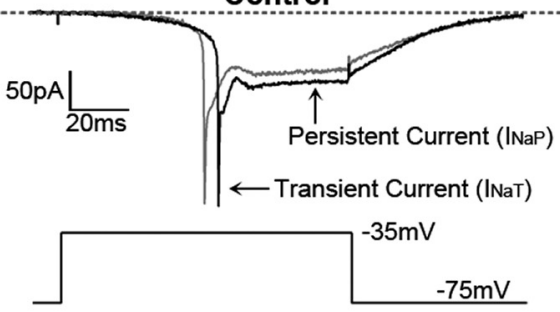

C

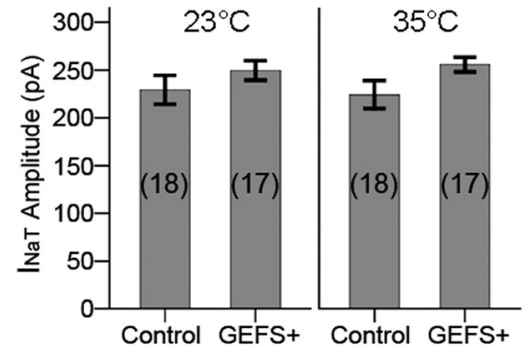

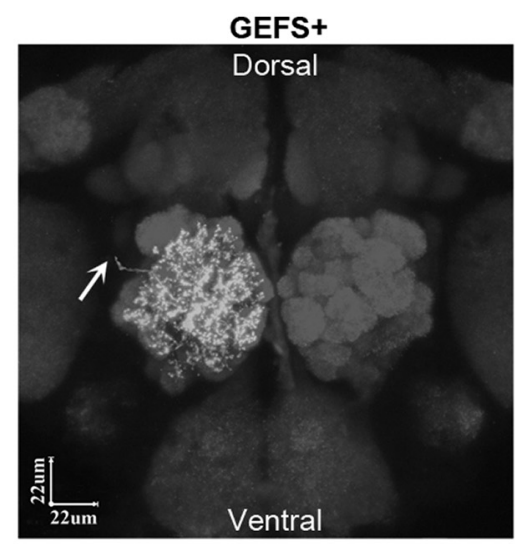

GEFS+
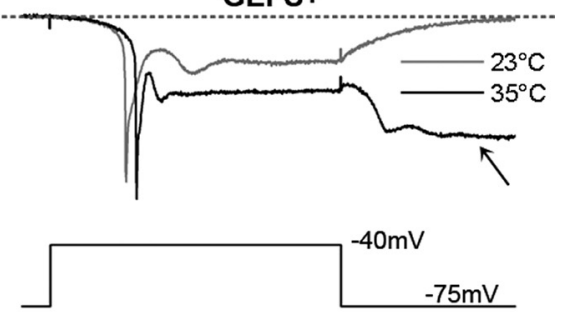

D

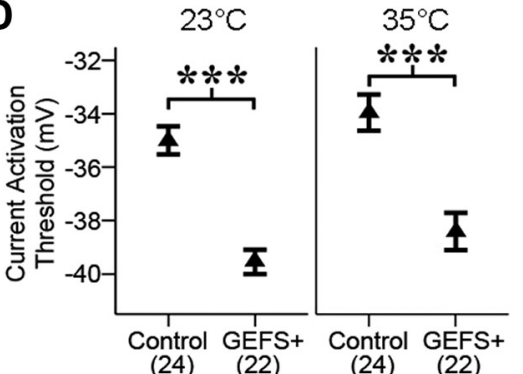

Figure 3. Sodium currents in GEFS + and control LNs. A, A LN in the dorsal lateral cluster in the antennal lobe, filled with biocytin during recording, in a control and GEFS + brain. Confocal images of the brains fixed and double stained with a fluorescently labeled secondary antibody to biocytin and Nc82 (anti-bruchtpilot antibody) are shown. Arrows indicate the LN cell body (control) or axon initial segment (GEFS+). The soma of the GEFS + cell was lost during pipette removal. $\boldsymbol{B}$, Depolarizing voltagestep-elicited sodium currents at room temperature $\left(23^{\circ} \mathrm{C}\right)$ and following heating of the recording solution to high temperature $\left(35^{\circ} \mathrm{C}\right) . I_{\mathrm{NaP}}$ in control LNs decayed to baseline after the pipette potential returned to $-75 \mathrm{mV}$, but it remained activated in the $\mathrm{GEFS}+\mathrm{LN}$ at $35^{\circ} \mathrm{C}$ (arrow). Sodium currents could not be clamped in either genotype. $C, I_{\mathrm{NaT}}$ amplitudes are no different in GEFS + and control LNs at 23 or $35^{\circ} \mathrm{C}$ (independent $t$ test, $23^{\circ} \mathrm{C}, p=0.28 ; 35^{\circ} \mathrm{C}, p=0.07$ ), and the amplitude did not change with temperature in either genotypes (paired $t$ test, control, $p=0.08 ; \mathrm{GEFS}+, p=0.20$ ). $\boldsymbol{D}$, The voltage step required to elicit the first inward sodium current is significantly more hyperpolarized in GEFS + than in control at both 23 and $35^{\circ} \mathrm{C}$. ${ }^{* * *} p<0.001$ (independent $t$ test). The current activation threshold did not change significantly with temperature in either genotype (paired $t$ test, control, $p=0.06$; GEFS,$+ p=0.14)$. Symbols and error bars represent mean \pm SEM.

\section{Alterations in evoked firing properties in GEFS + LNs at elevated temperature}

To investigate how the change in sodium currents affects LN excitability, we first examined firing properties in response to depolarizing current injections. To reduce the spontaneous activity in LNs, all cells were held at $-75 \mathrm{mV}$, and synaptic blockers PTX and curarine were added to the recording solution.

In both control and GEFS + LNs at room temperature, suprathreshold current injections evoked depolarizations capped by a train of small amplitude rapidly rising and decaying events termed spikelets (Fig. 5A, $B$ ). The spikelets, characteristic of action potentials recorded in the cell bodies of LNs, were blocked by TTX (data not shown) demonstrating their dependence on the activity of voltagegated sodium channels.
When the temperature was raised to $35^{\circ} \mathrm{C}$, trains of spikelets were maintained throughout the current step in control neurons. In contrast, spikelets in GEFS+ neurons often terminated before the end of the current step. Even more striking was the appearance of sustained depolarization after the current step that ranged in duration from $50 \mathrm{~ms}$ to $>10 \mathrm{~s}$ (Fig. $5 B$, arrow). The incidence of poststimulus depolarization in GEFS + LNs was $~ 10 \%$ at room temperature, and this was reversibly increased to $60 \%$ at $35^{\circ} \mathrm{C}$ consistent with the nondeactivating $I_{\mathrm{NaP}}$ seen in GEFS+ LNs at elevated temperature (Fig. 5C). The incidence of poststimulus depolarization was low in control LNs at both 23 and $35^{\circ} \mathrm{C}$ (Fig. 5C).

The spikelet frequency increased steadily with increasing current injection in control LNs at both 23 and $35^{\circ} \mathrm{C}$ (Fig. $5 A i)$. In contrast, the spikelet frequency declined in GEFS + LNs at higher input currents (Fig. 5Bi). In addition, even though the maximal firing frequency in GEFS + LNs was similar to control at room temperature, it was significantly and reversibly reduced, rather than increased as in control, when the temperature was elevated (Fig. 5D).

Together, these data indicate that the failure of the $I_{\mathrm{NaP}}$ to deactivate in response to normal membrane potential repolarization at elevated temperature results in prolonged poststimulus depolarization and reduced firing frequency in GEFS+ LNs at $35^{\circ} \mathrm{C}$.

\section{Alterations in spontaneous firing of GEFS + LNs at high temperature}

To determine whether there were differences between the two genotypes under more physiological conditions, spontaneous activity in LNs was monitored in the absence of synaptic current blockers and without application of hyperpolarizing holding current. At permissive temperature, both GEFS + and control LNs fire regular bursts of action potentials.

The standard response of control LNs to temperature change is illustrated in Figure $6 \mathrm{~A}$. The baseline burst frequency increased briefly when the temperature began to rise. As the temperature was elevated to $35^{\circ} \mathrm{C}$, the burst frequency decreased and then ceased. Normal burst firing resumed as the temperature returned to $23^{\circ} \mathrm{C}$. A similar change in burst frequency was seen in GEFS+ LNs at high temperature (Fig. 6D). However, as predicted from the changes observed in evoked firing, a significant fraction of the bursts in LNs progress into sustained membrane depolarizations at elevated temperatures (Fig. 6 Bii). A sustained depolarization is defined as an event (1) that is the same amplitude as the burst before heating, (2) that lacks spikelets, and (3) where the duration is longer than the mean burst duration ( $>95 \%$ confidence interval) at $23^{\circ} \mathrm{C}$. The duration of the sustained depolarizations in the 
A
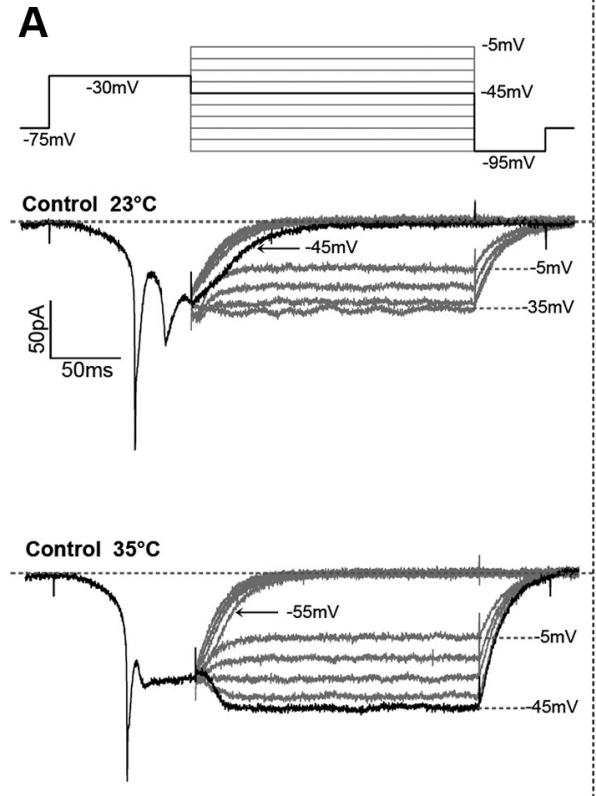

Ai Control

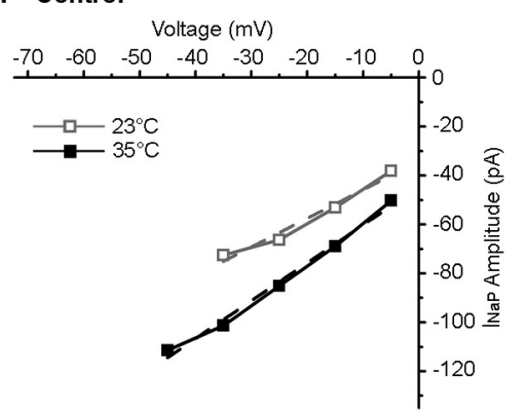

B

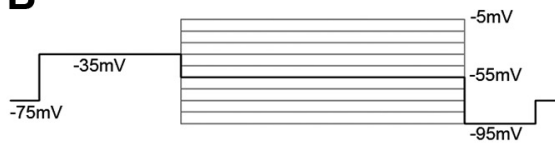

GEFS $+23^{\circ} \mathrm{C}$

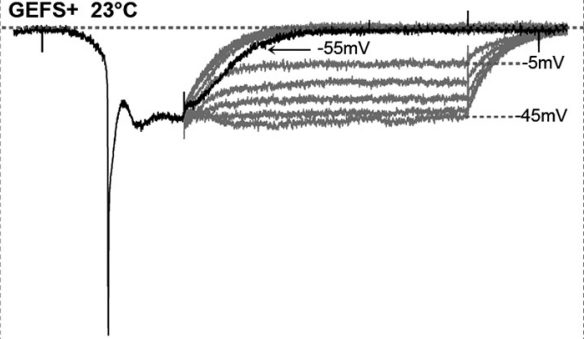

GEFS $+35^{\circ} \mathrm{C}$

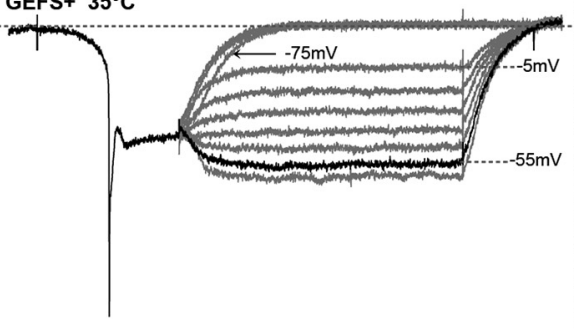

Bi GEFS+

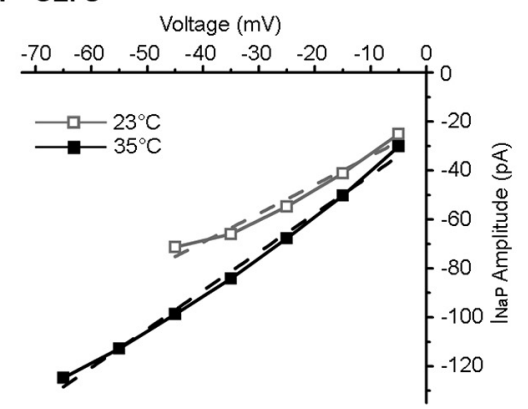

C
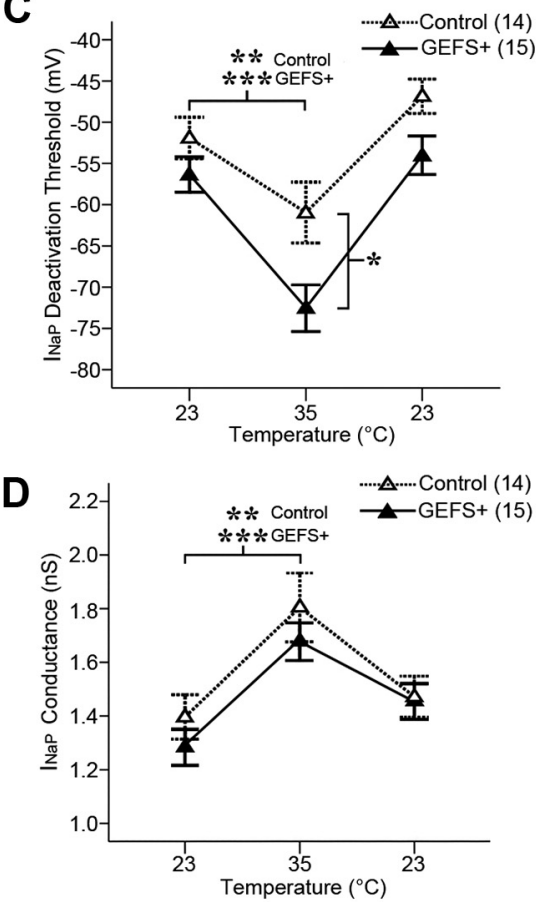

E

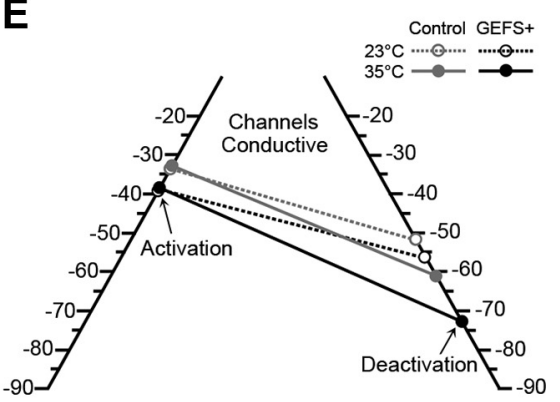

Figure 4. Temperature-induced hyperpolarizing shift in $I_{\text {Nap }}$ deactivation threshold is significantly larger in GEFS + LNs. $A, B$, Families of $I_{\text {Nap }}$ associated with the variable test step during the three-step voltage protocol illustrated in a typical control and GEFS $+\mathrm{LN}$ at 23 and $35^{\circ} \mathrm{C}$. Only one trace during the prepulse is shown for clarity. The first test potential that results in decay of the current back to baseline is defined as the current deactivation threshold voltage and is indicated by the arrow in each set of currents. When the temperature is raised from 23 to $35^{\circ} \mathrm{C}$, current deactivation voltage shifts from -45 to $-55 \mathrm{mV}$ in control and -55 to $-75 \mathrm{mV}$ in GEFS $+\mathrm{LNs}$. Ai, Bi, I-V curves representing $/_{\text {Nap }}$ amplitude measured during last $10 \mathrm{~ms}$ of the test pulse for each test potential from the traces shown above. Dashed lines are linear fits used to calculate the conductance of the $I_{\text {Nap. }} \mathrm{C}$, The deactivation voltage is more hyperpolarized at $35^{\circ} \mathrm{C}$ compared to $23^{\circ} \mathrm{C}$ in both control and GEFS + . However, the deactivation voltage is significantly more hyperpolarized in GEFS + than in control at $35^{\circ} \mathrm{C}$. D, Mean $/_{\text {NaP }}$ conductance reversibly increase at $35^{\circ} \mathrm{C}$ in both genotypes but was not different between GEFS + and control at $23^{\circ} \mathrm{C}$ (independent $t$ test, $p=0.29$ ) or $35^{\circ} \mathrm{C}(p=0.38) .{ }^{*} p<0.05$ (independent $t$ test); ${ }^{* *} p<0.01$, ${ }^{* * *} p<0.001$ (paired $t$ test). $E$, Average activation and deactivation threshold voltage of sodium currents in control and GEFS + at room temperature and elevated temperature. GEFS + LNs have a wider voltage range over which sodium channels are conductive. Symbols and error bars represent mean \pm SEM.

mutant neurons measured at half amplitude ranged from $315 \mathrm{~ms}$ to $>16 \mathrm{~s}$.

At permissive temperatures, the sustained membrane depolarizations in GEFS + occur at low frequency and represent only a small percentage of total membrane depolarizations (bursts plus sustained depolarizations). In contrast, when the temperature was raised to $35^{\circ} \mathrm{C}$, the frequency increased almost threefold (Fig. $6 C$ ) and the percentage increased from 1.3 to $23 \%$ (Fig. 6D). When the temperature returned to $23^{\circ} \mathrm{C}$, the duration of depolarization returned to the level seen before heating. In control LNs, sustained depolarizations were not observed at $23^{\circ} \mathrm{C}$ and this was not significantly increased at $35^{\circ} \mathrm{C}$ (Fig. 6C,D).

The spikelet frequency (number of spikelets/duration of depolarization) was calculated for both genotypes. In GEFS + LNs, the spikelet frequency decreased significantly at high temperatures (Fig. 6E). In contrast, control LNs generated spikelet capped bursts at $35^{\circ} \mathrm{C}$ and the spikelet frequency increased at elevated temperatures compared to room temperature, consistent with the evoked activity (Fig. 6E). In addition, spikelet threshold was $5 \mathrm{mV}$ more hyperpolarized in GEFS + compared to control at both 23 and $35^{\circ} \mathrm{C}$ (Fig. $6 F$ ), consistent with the difference in sodium current activation threshold (Fig. $3 D$ ).

The appearance of sustained depolarizations at high temperature is consistent with the poststimulus depolarization observed in evoked firing. These data indicate that the more negative deactivation voltage of the $I_{\mathrm{NaP}}$ in GEFS + LNs at elevated temperature results in sustained membrane depolarization and reduced repetitive firing.

Blockade of GABA receptors increases sensitivity to heatinduced seizures in GEFS+ flies

Reduced repetitive firing in GEFS + GABAergic neurons suggests that a decrease in inhibitory activity at high temperature contributes to the heat-induced seizure phenotype in GEFS + . This pre- 

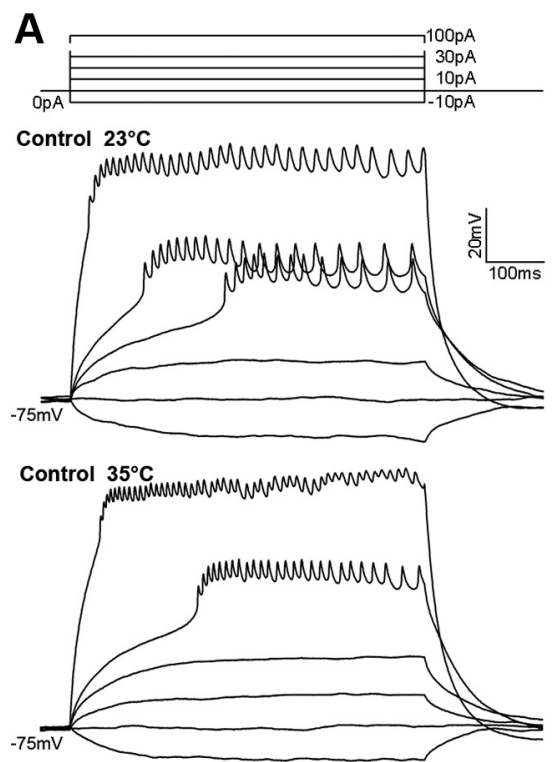

Ai Control

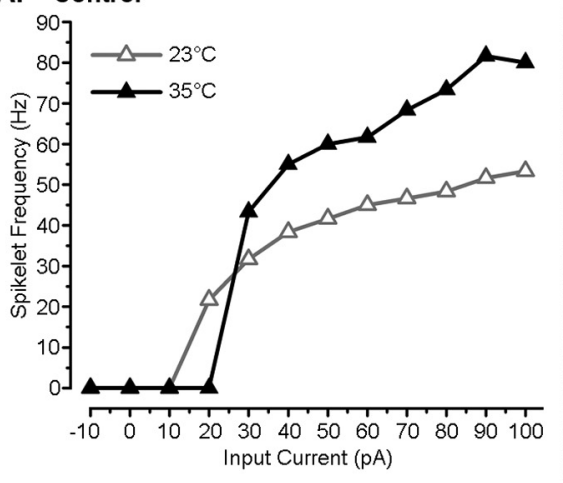

B

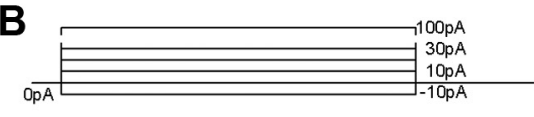

GEFS $+23^{\circ} \mathrm{C}$

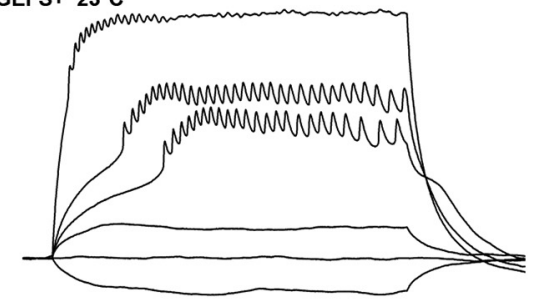

GEFS $+35^{\circ} \mathrm{C}$

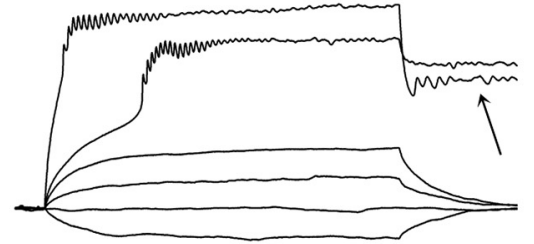

Bi GEFS+

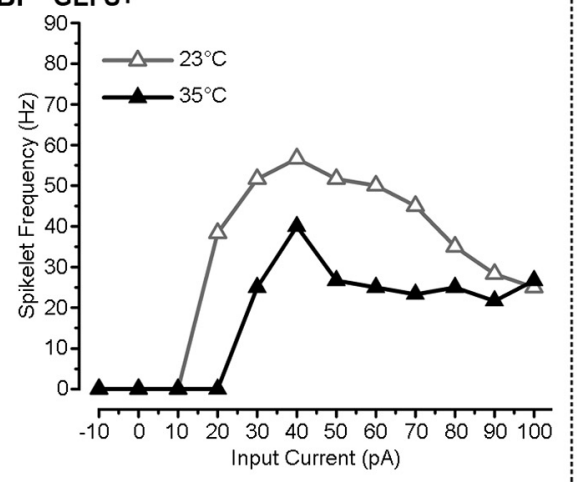

C

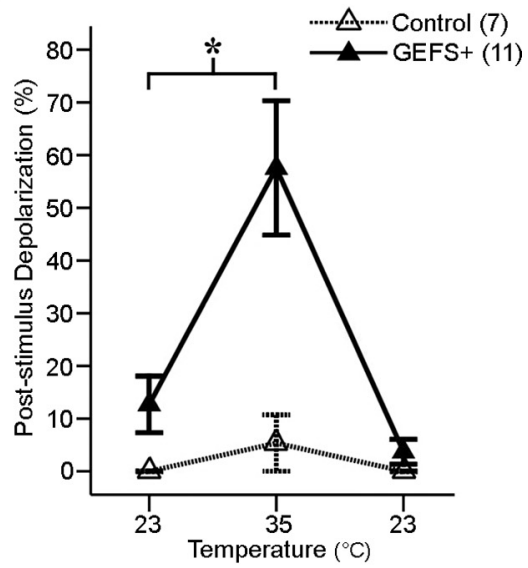

D

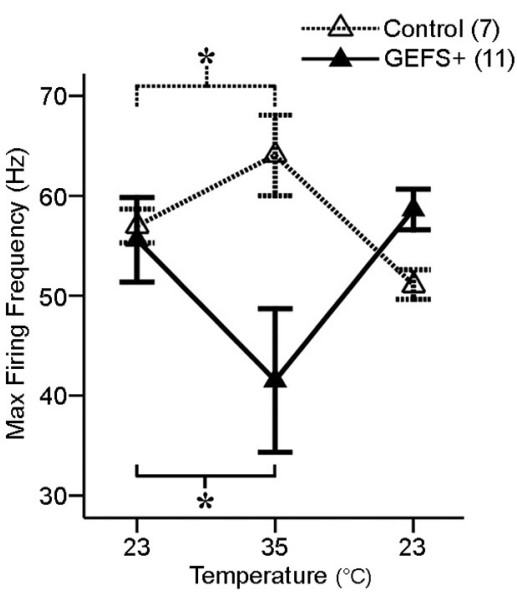

Figure 5. Alterations in evoked firing properties of GEFS + LNs at elevated temperature. $A, B$, Representative trains of spikelets recorded from control and GEFS $+\mathrm{LNs}$ at 23 and $35^{\circ} C$ evoked by the stimulus protocol illustrated. $\boldsymbol{A i}, \boldsymbol{B i}$, Spikelet frequency plotted as a function of injected current for $\mathrm{LN}$ s shown above. $\boldsymbol{C}$, The incidence of poststimulus depolarization is defined as the percentage of traces in which there was a poststimulus depolarization that lasted for at least $50 \mathrm{~ms}$ in each $L N$. The incidence of GEFS + LNs showing poststimulus depolarizations increased significantly at $35^{\circ} \mathrm{C}$ compared to $23^{\circ} \mathrm{C}$ (paired $t$ test, ${ }^{*} p<0.05$ ). In contrast, the incidence of poststimulus depolarization was low in control LNs and did not increase significantly at elevated temperature $(p=0.36)$. $D$, Raising the temperature from 23 to $35^{\circ} \mathrm{C}$ resulted in a significant increase in the maximal firing frequency in control LNs and a significant decrease in GEFS + LNs. ${ }^{*} p<0.05$ (paired $t$ test). Symbols and error bars represent mean \pm SEM.

dicts that GEFS + flies should be more sensitive to the $\mathrm{GABA}_{\mathrm{A}} \mathrm{R}$ antagonist PTX, an established chemiconvulsant in Drosophila as well as mouse (Stilwell et al., 2006). Adult males were fed sucrose solutions containing different concentrations of PTX and seizure probability was assessed within $5-30 \mathrm{~min}$. At $37^{\circ} \mathrm{C}$, GEFS + flies fed sucrose without PTX displayed very low seizure activity after 2 min (Fig. 7A). In contrast to seizures at $40^{\circ} \mathrm{C}$, these seizures were short and infrequent with flies seizing on average for only a short fraction of the heating period (Fig. 7B). GEFS+ seizure probability and time spent seizing when exposed to $37^{\circ} \mathrm{C}$ increased in a PTX dose-dependent manner (Fig. $7 A, B$ ). Control flies fed the highest dose of PTX used in these experiments did not show any seizure behavior at $37^{\circ} \mathrm{C}$ (Fig. $\left.7 A, B\right)$. These results support the hypothesis that the GEFS + seizure phenotype arises from reduced inhibitory activity at elevated temperatures.

\section{Discussion}

Genotype-to-phenotype mapping of human sodium channel disease in Drosophila

To create a human epilepsy model in Drosophila, we used homologous recombination to introduce a specific disease-causing mutation into the equivalent Drosophila gene. Knock-in of the human K1270T GEFS+ SCN1A mutation into the Drosophila para sodium channel gene results in adult flies that exhibit seizures triggered by high temperature. This is consistent with the effect of the K1270T mutation in humans. GEFS+ patients heterozygous for this mutation have febrile seizures that can persist beyond childhood (Abou-Khalil et al., 2001). The temperature-sensitive seizure phenotype in mutant flies is semidominant: heterozygotes exhibit seizure activity, but it is reduced compared to homozygotes. Although patients homozygous for the mutation have not been reported, more severe phenotypes are expected based on the observation in the fly model.

The seizure phenotype in GEFS + flies is distinct from phenotypes resulting from random para sodium channel mutations isolated previously in classic forward genetic screens. One class of para mutants causes a temperature-sensitive paralysis (Suzuki et al., 1971; Grigliatti et al., 1973; Siddiqi and Benzer, 1976), whereas a second class causes bang-sensitive seizure (Jan and Jan, 1978; Ganetzky and Wu, 1982; Parker et al., 2011). One of the previously defined para mutations, G1517R, is positionally orthologous to the G1306V mutation in the human SCN4A skeletal muscle sodium channel gene. This mutation causes a dominant 


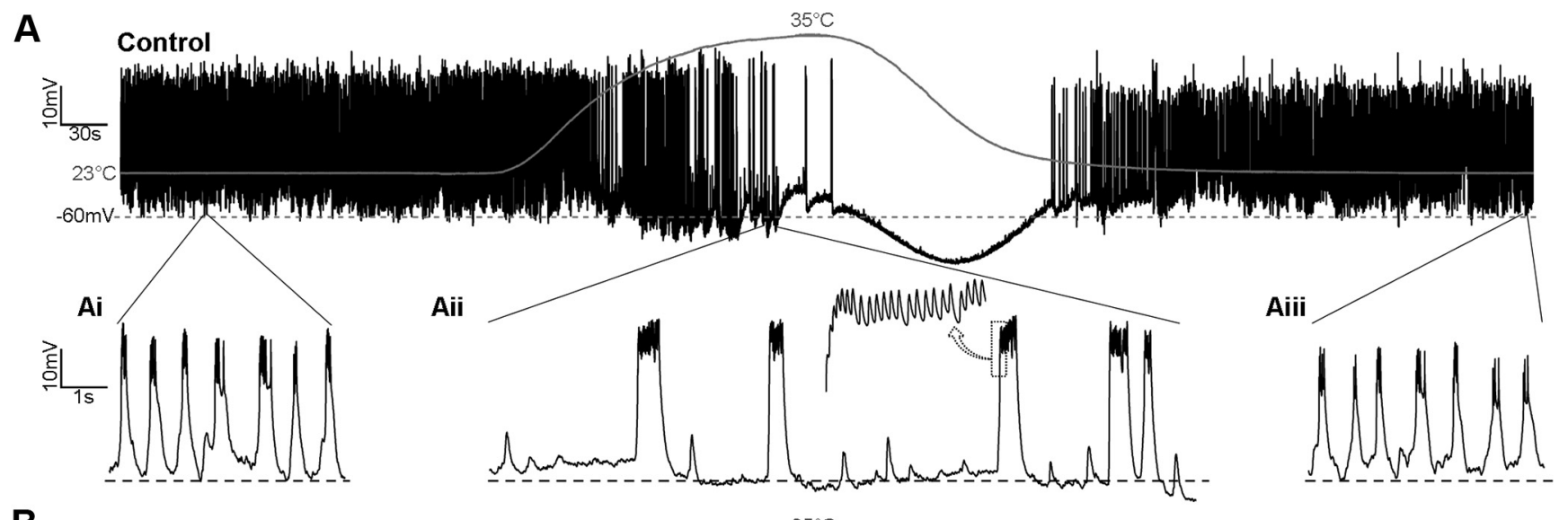

B
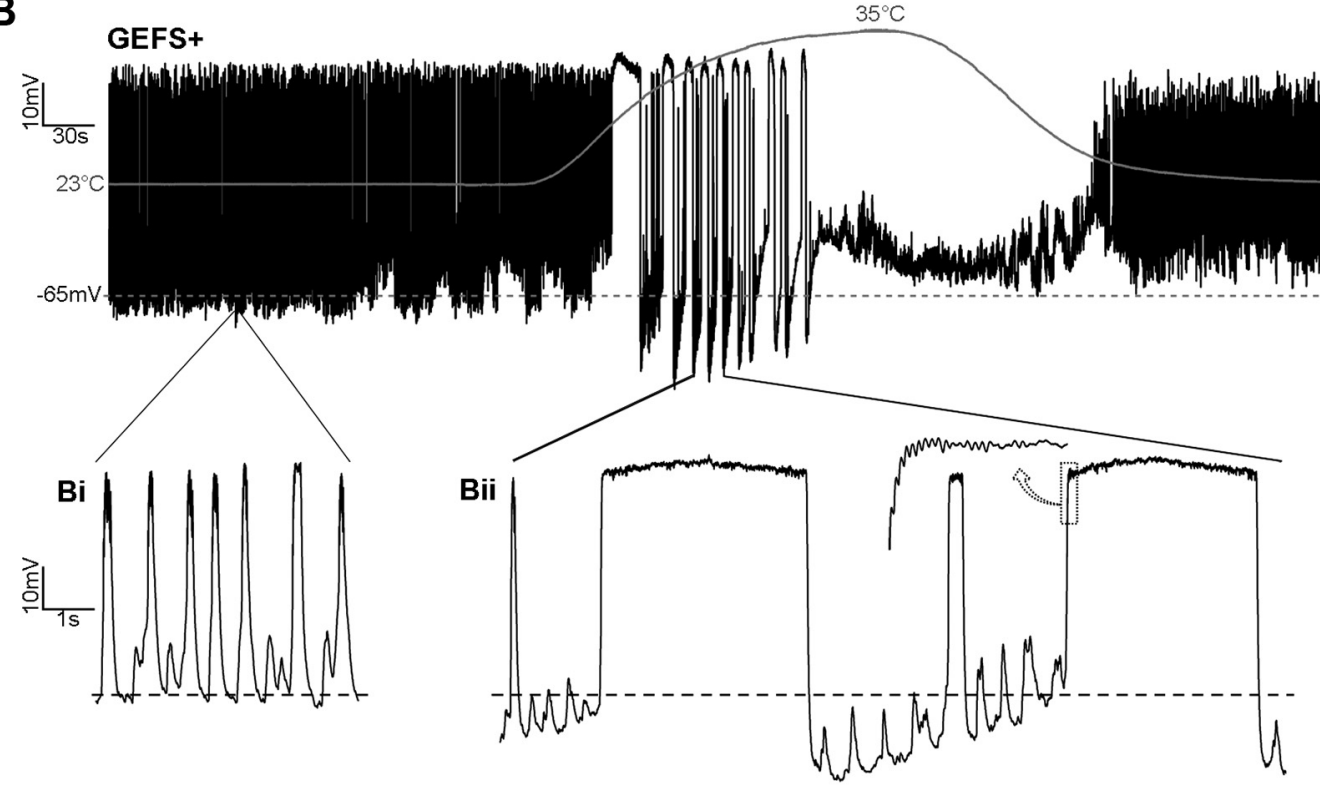

C
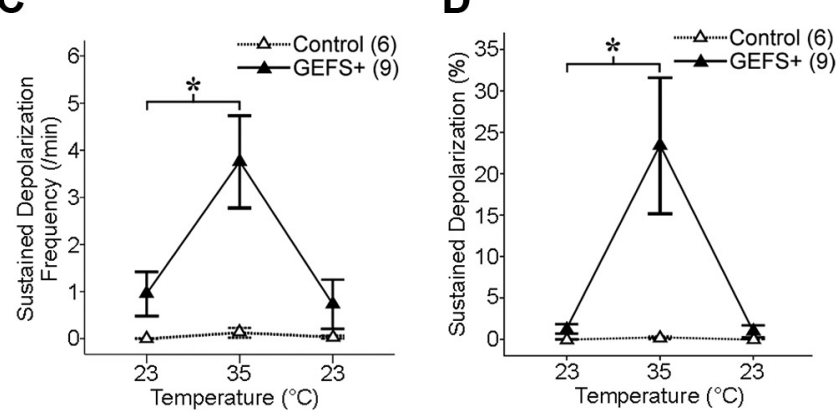

E

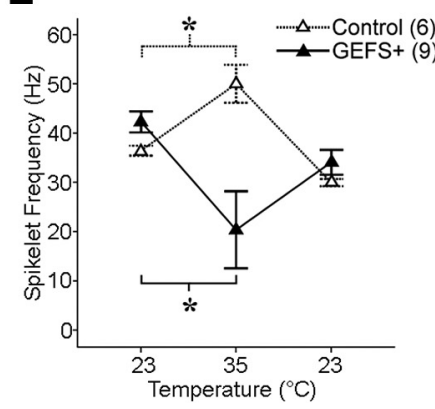

$\mathbf{F}$

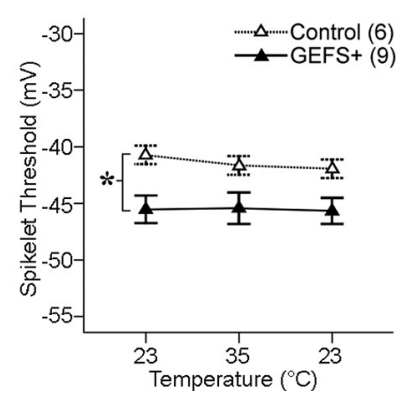

Figure 6. Appearance of sustained depolarizations without spikelets in GEFS + LNs at high temperature. $A, B$, Continuous recordings of spontaneous activity in control and GEFS + LNs as the temperature is raised and lowered as indicated by the overlay of the temperature probe recording. Ai-Aiii, Bi-Biii, Traces on expanded time scales from the regions indicated. In both genotypes, the regular burst firing frequency gradually decreases as the temperature increases. Before cessation of firing, activity in GEFS + LNs at high temperature is characterized by the appearance of sustained depolarizations without spikelets that are not observed in control LNs. Normal burst firing resumes in both genotypes when the bath is returned to room temperature. $C$, The frequency of sustained membrane depolarizations in GEFS $+\mathrm{LNs}$ is increased at high temperature. These events were counted at $23^{\circ} \mathrm{C}\left(5\right.$ min before heating), at high temperature $\left(30-35^{\circ} \mathrm{C}\right)$, and following return to room temperature ( $0-5 \mathrm{~min}$ after return to room temperature). $\boldsymbol{D}$, The percentage of membrane depolarizations that were sustained increased at $35^{\circ} \mathrm{C}$ in GEFS.$+ \boldsymbol{E}$, Spikelet frequency increased in control LNs, but decreased in GEFS $+\mathrm{LNs}$ at $35^{\circ} \mathrm{C}$ compared to $23^{\circ} \mathrm{C}$. F, The spikelet threshold is more hyperpolarized in GEFS + than in control LNs at both 23 and $35^{\circ} \mathrm{C}$ (independent $t$ test, $p<0.05$ ). The spikelet threshold did not change as a function of temperature in either GEFS + (paired $t$ test, $p=0.95$ ) or control LNs $\left(p=0.23\right.$ ). ${ }^{*} p<0.05$ (paired $t$ test). Symbols and error bars represent mean \pm SEM.

cold-sensitive paralytic phenotype in Drosophila (Lindsay et al., 2008), reminiscent of the cold-sensitive disorder paramyotonia congenita associated with the human mutation. These findings combined with our results suggest a remarkable and specific conservation of genotype-to-phenotype mapping of human sodium channel disease in Drosophila.
Decreased inhibitory activity contributing to seizures in GEFS+ mutants

Studies in knock-in mouse models of a GEFS+ (R1648H) and a DS (R1407X) mutation report reduced activity in inhibitory but not excitatory neurons, suggesting that decreased inhibition contributes to seizure generation in patients with these disorders 

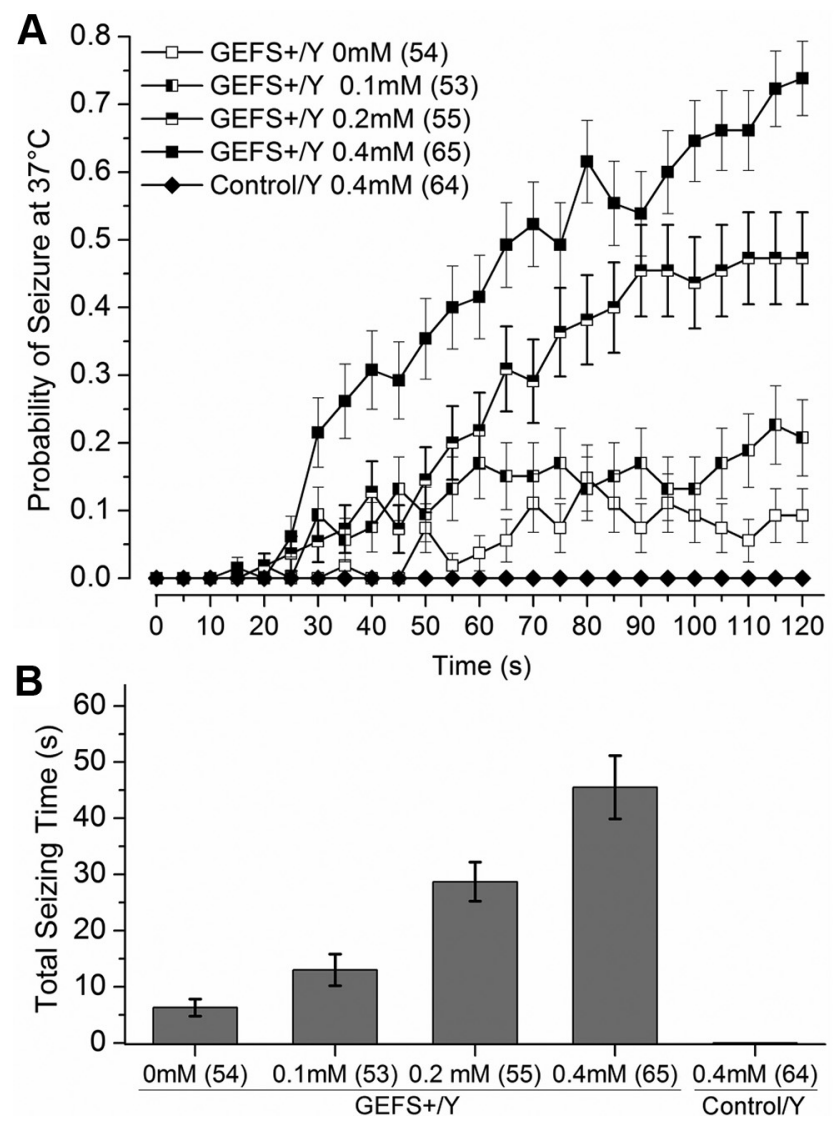

Figure 7. PTX increases GEFS + sensitivity to heat-induced seizures. $A$, Flies were fed sucrose with indicated concentration of PTX and tested for seizure susceptibility within $30 \mathrm{~min}$. The probability of seizing in GEFS + flies after 2 min at $37^{\circ} \mathrm{C}$ increases with PTX concentration $(p<0.001$, one-way ANOVA). Control flies fed the maximal dose of PTX ( $0.4 \mathrm{~mm})$ did not show seizure behavior at $37^{\circ} \mathrm{C} . \boldsymbol{B}$, In GEFS + flies, the time spent seizing during the 2 min heat exposure increases with PTX concentration ( $p<0.001$, one-way ANOVA). Symbols and error bars represent mean $\pm \mathrm{SEM}$.

(Ogiwara et al., 2007; Escayg and Goldin, 2010; Martin et al., 2010). Does a GEFS + mutation also differentially alter activity in inhibitory versus excitatory neurons in Drosophila? Our data demonstrate that the GEFS + K1270T mutation results in reduced repetitive firing in LNs, a population of GABAergic interneurons in adult fly brain, at elevated temperature. Preliminary analysis of excitatory cholinergic projection neurons did not reveal any evidence of poststimulus depolarization in evoked firing at either $23^{\circ} \mathrm{C}(0$ of 15$)$ or $35^{\circ} \mathrm{C}(0$ of 11$)$. This suggests that the GEFS + mutation also has a more pronounced effect on inhibitory versus excitatory neurons in Drosophila. There is evidence that specificity in the mouse model arises from differential expression of SCN1A encoded sodium channels in these two populations (Ogiwara et al., 2007; Lorincz and Nusser, 2008; Wimmer et al., 2010). Although there is only a single sodium channel gene in Drosophila, there are splice variants with distinct functional properties (Lin et al., 2009; Marley and Baines, 2011) that could be differentially expressed in excitatory versus inhibitory neurons.

From the knock-in mouse models it seems likely that decreased activity in central inhibitory circuits at room temperature contributes to generation of seizures in patients with $\mathrm{R} 1648 \mathrm{H}$ and R1047X SCN1A mutations (Ogiwara et al., 2007; Martin et al., 2010). However, in the absence of recording at high temperature, it is not clear whether additional temperature-dependent changes in activity contribute to the febrile seizure phenotype. Analysis of the K1270T mutation in Drosophila revealed reduced repetitive firing in GABAergic neurons, suggesting a decrease in inhibitory activity, but the changes in neuronal firing were primarily linked to elevation in temperature. The GEFS + flies were also more sensitive to heat-induced seizures after feeding with the $G_{A B A} R$ antagonist PTX, an established chemiconvulsant in animal models (Stilwell et al., 2006). These results support the hypothesis that a temperature-dependent decrease in inhibitory activity can contribute to the heat-induced seizure phenotype in GEFS + .

\section{A new mechanism underlying temperature sensitive seizures} In the mouse model, knock-in of the $\mathrm{R} 1648 \mathrm{H}$ mutation, located in transmembrane segment 4 in domain IV (S4,DIV) of the sodium channel, resulted in a decrease in firing frequency in GABAergic neurons at room temperature. This was caused by constitutive changes in the $I_{\mathrm{NaT}}$ including a decrease in amplitude, slower recovery from inactivation, and greater usedependent inactivation (Tang et al., 2009; Martin et al., 2010). In contrast, the most prominent alteration observed in our fly model with the K1270T mutation located in segment 2 in domain III (S2,DIII) was an increase in the hyperpolarizing shift in $I_{\mathrm{NaP}}$ deactivation when then temperature was elevated. The failure of the $I_{\mathrm{NaP}}$ to deactivate contributes to sustained depolarizations and reduced firing frequency in GEFS $+\mathrm{LNs}$ at $35^{\circ} \mathrm{C}$. There was also a negative shift in the sodium current activation threshold at both room and elevated temperature in GEFS + LNs. While this is likely to contribute to increased firing at room temperature, it could also lead to reduced firing and sustained depolarizations at high temperature when deactivation is impaired. These data support the hypothesis that GEFS+-causing SCN1A mutations located in different regions of the gene result in distinct alterations in channel function. Analysis of neuronal sodium currents in other GEFS + mutants, at permissive and elevated temperatures, will be crucial in developing therapies targeted to particular mutations.

Temperature is known to influence kinetics of ion channel gating, but there is relatively little information about the effect of temperature on the voltage dependence of channel gating. How might temperature sensitivity in voltage-dependent deactivation arise? In Drosophila, there is convincing evidence that a single sodium channel subtype can switch between different gating modes to generate both a transient and persistent $\mathrm{Na}^{+}$current (Liu et al., 2004; Lin et al., 2009). Single-channel studies in mammalian neurons indicate that a persistent sodium current can arise from late opening of a channel or an individual channel that fails to inactivate and produces a burst of openings during maintained depolarization (Alzheimer et al., 1993; Stafstrom, 2007). Our data demonstrate that repolarization to more negative voltages is required to deactivate the $I_{\mathrm{NaP}}$ at elevated temperatures, even in control neurons. This suggests that the wild-type sodium channel protein undergoes a temperature-dependent structural alteration that requires more negative voltage to close the channels still active during a maintained depolarization. Since the K1270T mutation results in a more negative shift in the deactivation threshold at high temperature, this suggests that the mutation identifies a possible position in S2,DIII associated with the temperature-sensitive closing transition of channels underlying the $I_{\mathrm{NaP}}$.

Given the small but significant change in gating seen at high temperature in control neurons, we asked whether control flies develop seizure-like activity under more extreme conditions. At $41^{\circ} \mathrm{C}$, some of the control flies displayed seizures, and the seizure 
probability increased further at $42^{\circ} \mathrm{C}$ (data not shown). Thus, it appears that temperature-induced shifts in voltage dependence of sodium current deactivation could contribute to generation of seizures even in control flies at elevated temperatures. A recent study reported a hyperpolarizing shift in activation voltage of sodium currents in HEK293 cells expressing wild-type Nav1.2 channels when the temperature was raised from 37 to $41^{\circ} \mathrm{C}$ (Thomas et al., 2009). Computer simulations indicated this would increase neuronal excitability at high temperature. Together, these data suggest that temperature-induced changes in voltage dependence of channel gating could contribute to genesis of febrile seizures even in the absence of known seizureassociated mutations.

\section{Genetic screen for modifiers that regulate the seizures}

GEFS + in humans is classified as an autosomal dominant syndrome. However, in GEFS + patients carrying a single mutant allele, the seizure phenotype can vary considerably (Wallace et al., 1998). This is true in the case of the K1270T GEFS + mutation (Abou-Khalil et al., 2001). Pedigree analysis in a large family revealed 7 affected family members with febrile seizures only, 11 with febrile seizures beyond 6 years of age, and 12 with complex febrile seizures. In addition, one individual carrying this mutation had no evidence of seizures. Such results hint at a complex genetic architecture of this disease, influenced by genetic modifiers and/or environmental factors. However, evaluating the contribution of each is difficult in light of enormous variation in genetic background and living conditions between individuals.

In the fly model, the effect of the K1270T mutation on seizure phenotype is dose dependent. Similar to human GEFS + patients carrying one mutant allele, the heterozygous GEFS + flies also exhibit differences in seizure severity. In response to increased temperature, some heterozygous flies display persistent seizure, while others do not seize at all under the same experimental conditions. Therefore, it will be interesting to rear GEFS mutants under well-controlled environmental conditions, inbreed flies that exhibit persistent seizures, and compare these to inbred flies that do not seizure at elevated temperature. If this results in two GEFS + lines that breed true with respect to their seizure severity, it should be possible to identify the genetic modifiers affecting manifestation of the seizure phenotype using deep sequencing methods (Blumenstiel et al., 2009). Thus, this strategy has the potential to identify novel gene targets for development of therapies aimed at ameliorating seizure severity in GEFS + patients.

In summary, the congruence of the genotype-to-phenotype map between flies and human paves the way for using knock-in Drosophila models to study the mechanisms underlying complex human genetic diseases. In addition, the ability to combine the power of low-cost, large-scale forward genetic screens with the knock-in strategy will be extremely useful in identifying novel genes involved in regulating human disease.

\section{References}

Abou-Khalil B, Ge Q, Desai R, Ryther R, Bazyk A, Bailey R, Haines JL, Sutcliffe JS, George AL Jr (2001) Partial and generalized epilepsy with febrile seizures plus and a novel SCN1A mutation. Neurology 57:2265-2272.

Alzheimer C, Schwindt PC, Crill WE (1993) Modal gating of Na + channels as a mechanism of persistent $\mathrm{Na}+$ current in pyramidal neurons from rat and cat sensorimotor cortex. J Neurosci 13:660-673.

Blumenstiel JP, Noll AC, Griffiths JA, Perera AG, Walton KN, Gilliland WD, Hawley RS, Staehling-Hampton K (2009) Identification of EMS-induced mutations in Drosophila melanogaster by whole-genome sequencing. Genetics 182:25-32.

Catterall WA, Kalume F, Oakley JC (2010) NaV1.1 channels and epilepsy. J Physiol 588:1849-1859.
Chou YH, Spletter ML, Yaksi E, Leong JC, Wilson RI, Luo L (2010) Diversity and wiring variability of olfactory local interneurons in the Drosophila antennal lobe. Nat Neurosci 13:439-449.

Claes LR, Deprez L, Suls A, Baets J, Smets K, Van Dyck T, Deconinck T, Jordanova A, De Jonghe P (2009) The SCN1A variant database: a novel research and diagnostic tool. Hum Mutat 30:E904-E920.

Escayg A, Goldin AL (2010) Sodium channel SCN1A and epilepsy: mutations and mechanisms. Epilepsia 51:1650-1658.

Fergestad T, Bostwick B, Ganetzky B (2006) Metabolic disruption in Drosophila bang-sensitive seizure mutants. Genetics 173:1357-1364.

Ganetzky B, Wu CF (1982) Indirect suppression involving behavioral mutants with altered nerve excitability in Drosophila melanogaster. Genetics 100:597-614.

Grigliatti TA, Hall L, Rosenbluth R, Suzuki DT (1973) Temperaturesensitive mutations in Drosophila melanogaster. XIV. A selection of immobile adults. Mol Gen Genet 120:107-114.

Gu H, O'Dowd DK (2006) Cholinergic synaptic transmission in adult Drosophila Kenyon cells in situ. J Neurosci 26:265-272.

Gu H, O’Dowd DK (2007) Whole cell recordings from brain of adult Drosophila. J Vis $\operatorname{Exp} 6: 248$.

Jan YN, Jan LY (1978) Genetic dissection of short-term and long-term facilitation at the Drosophila neuromuscular junction. Proc Natl Acad Sci U S A 75:515-519.

Lin WH, Wright DE, Muraro NI, Baines RA (2009) Alternative splicing in the voltage-gated sodium channel DmNav regulates activation, inactivation, and persistent current. J Neurophysiol 102:1994-2006.

Lindsay HA, Baines R, ffrench-Constant R, Lilley K, Jacobs HT, O’Dell KM (2008) The dominant cold-sensitive Out-cold mutants of Drosophila melanogaster have novel missense mutations in the voltage-gated sodium channel gene paralytic. Genetics 180:873-884.

Liu Z, Song W, Dong K (2004) Persistent tetrodotoxin-sensitive sodium current resulting from U-to-C RNA editing of an insect sodium channel. Proc Natl Acad Sci U S A 101:11862-11867.

Lorincz A, Nusser Z (2008) Cell-type-dependent molecular composition of the axon initial segment. J Neurosci 28:14329-14340.

Lossin C (2009) A catalog of SCN1A variants. Brain Dev 31:114-130.

Lossin C, Wang DW, Rhodes TH, Vanoye CG, George AL Jr (2002) Molecular basis of an inherited epilepsy. Neuron 34:877-884.

Lossin C, Rhodes TH, Desai RR, Vanoye CG, Wang D, Carniciu S, Devinsky O, George AL Jr (2003) Epilepsy-associated dysfunction in the voltagegated neuronal sodium channel SCN1A. J Neurosci 23:11289-11295.

Maggert KA, Gong WJ, Golic KG (2008) Methods for homologous recombination in Drosophila. Methods Mol Biol 420:155-174.

Marley R, Baines RA (2011) Increased persistent Na+ current contributes to seizure in the slamdance bang-sensitive Drosophila mutant. J Neurophysiol 106:18-29.

Martin MS, Dutt K, Papale LA, Dubé CM, Dutton SB, de Haan G, Shankar A, Tufik S, Meisler MH, Baram TZ, Goldin AL, Escayg A (2010) Altered function of the SCN1A voltage-gated sodium channel leads to gammaaminobutyric acid-ergic (GABAergic) interneuron abnormalities. J Biol Chem 285:9823-9834.

Ogiwara I, Miyamoto H, Morita N, Atapour N, Mazaki E, Inoue I, Takeuchi T, Itohara S, Yanagawa Y, Obata K, Furuichi T, Hensch TK, Yamakawa K (2007) Nav1.1 localizes to axons of parvalbumin-positive inhibitory interneurons: a circuit basis for epileptic seizures in mice carrying an Scnla gene mutation. J Neurosci 27:5903-5914.

Parker L, Padilla M, Du Y, Dong K, Tanouye MA (2011) Drosophila as a model for epilepsy: bss is a gain-of-function mutation in the para sodium channel gene that leads to seizures. Genetics 187:523-534.

Pavlidis P, Ramaswami M, Tanouye MA (1994) The Drosophila easily shocked gene: a mutation in a phospholipid synthetic pathway causes seizure, neuronal failure, and paralysis. Cell 79:23-33.

Rong YS, Golic KG (2000) Gene targeting by homologous recombination in Drosophila. Science 288:2013-2018.

Rong YS, Titen SW, Xie HB, Golic MM, Bastiani M, Bandyopadhyay P, Olivera BM, Brodsky M, Rubin GM, Golic KG (2002) Targeted mutagenesis by homologous recombination in $D$. melanogaster. Genes Dev 16:1568-1581.

Royden CS, Pirrotta V, Jan LY (1987) The tko locus, site of a behavioral mutation in D. melanogaster, codes for a protein homologous to prokaryotic ribosomal protein S12. Cell 51:165-173.

Scheffer IE, Berkovic SF (1997) Generalized epilepsy with febrile seizures 
plus. A genetic disorder with heterogeneous clinical phenotypes. Brain 120:479-490.

Siddiqi O, Benzer S (1976) Neurophysiological defects in temperaturesensitive paralytic mutants of Drosophila melanogaster. Proc Natl Acad Sci U S A 73:3253-3257.

Song J, Tanouye MA (2008) From bench to drug: human seizure modeling using Drosophila. Prog Neurobiol 84:182-191.

Song J, Hu J, Tanouye M (2007) Seizure suppression by top1 mutations in Drosophila. J Neurosci 218:1015-1022.

Song J, Parker L, Hormozi L, Tanouye MA (2008) DNA topoisomerase I inhibitors ameliorate seizure-like behaviors and paralysis in a Drosophila model of epilepsy. Neuroscience 156:722-728.

Staber CJ, Gell S, Jepson JE, Reenan RA (2011) Perturbing A-to-I RNA editing using genetics and homologous recombination. Methods Mol Biol 718:41-73.

Stafstrom CE (2007) Persistent sodium current and its role in epilepsy. Epilepsy Curr 7:15-22.

Stilwell GE, Saraswati S, Littleton JT, Chouinard SW (2006) Development of a Drosophila seizure model for in vivo high-throughput drug screening. Eur J Neurosci 24:2211-2222.

Suzuki DT, Grigliatti T, Williamson R (1971) Temperature-sensitive muta- tions in Drosophila melanogaster. VII. A mutation (para-ts) causing reversible adult paralysis. Proc Natl Acad Sci U S A 68:890-893.

Tang B, Dutt K, Papale L, Rusconi R, Shankar A, Hunter J, Tufik S, Yu FH, Catterall WA, Mantegazza M, Goldin AL, Escayg A (2009) A BAC transgenic mouse model reveals neuron subtype-specific effects of a generalized epilepsy with febrile seizures plus (GEFS +) mutation. Neurobiol Dis 35:91-102.

Thomas EA, Hawkins RJ, Richards KL, Xu R, Gazina EV, Petrou S (2009) Heat opens axon initial segment sodium channels: a febrile seizure mechanism? Ann Neurol 66:219-226.

Wallace RH, Wang DW, Singh R, Scheffer IE, George AL Jr, Phillips HA, Saar K, Reis A, Johnson EW, Sutherland GR, Berkovic SF, Mulley JC (1998) Febrile seizures and generalized epilepsy associated with a mutation in the $\mathrm{Na}+$-channel betal subunit gene SCN1B. Nat Genet 19:366-370.

Wimmer VC, Reid CA, Mitchell S, Richards KL, Scaf BB, Leaw BT, Hill EL, Royeck M, Horstmann MT, Cromer BA, Davies PJ, Xu R, Lerche H, Berkovic SF, Beck H, Petrou S (2010) Axon initial segment dysfunction in a mouse model of genetic epilepsy with febrile seizures plus. J Clin Invest 120:2661-2671.

Zhang H, Tan J, Reynolds E, Kuebler D, Faulhaber S, Tanouye M (2002) The Drosophila slamdance gene: a mutation in an aminopeptidase can cause seizure, paralysis and neuronal failure. Genetics 162:1283-1299. 